\title{
Author Index Vol. 17, No. 3-4,1997
}

Aliotta,G. 241 Andreae, L. 359 Angeletti, L.R. 228 Angelopoulos, N. 214 Antonello,A. 248

Bellini, L. 318 Better, O.S. 392 Bisaccia, C. 318 Bonfante,L. 248 Bonomini, V. 274 Bordin,V. 248

Calò, L. 203,248 Cameron, J.S. 347 Campieri,C. 274 Cavarra,B. 228

D’Angelo,A. 248

Dal Canton, A. 282

Dal Canton, I. 282

Dardioti,V. 214

De Santo, D. 318

De Santo, L.S. 318

De Santo, N.G. 201,241,261,

318 De Santo, R.M. 318 De Weerdt, D.L. 252 DeBroe,M.E. 252 Diamandopoulos, A.A. 222, 304

Eftychiadis, A.C. 217 Eknoyan, G. 201,261

Favaro,S. 248 Fellner,S.K. 289 Fine,L.G. 359 Frosio-Roncalli, M. 282 Fukushima, M. 382

Gottschalk, C.W. 289

Hicks, J. 347 Hierholzer, J. 369 Hierholzer, K. 369

Inoue, T. 382 lorio, L. 286 Ishimura, E. 382

Marketos, S.G. 201,204,205 Massry,S.G. 201,233 Mezzogiorno, A. 269 Mezzogiorno, V. 269

Morii,H. 382 Morita,A. 382

Nacca,R.G. 286 Nagy,J. 387 Níshii,Y. 382

Peitzman, S.J. 299 Piomelli, D. 241 Pollio,A. 241

Poulakou-Rebelakou, E. 209 Purkerson, M.L. 340

248

Richet, G. 315 Rippa-Bonati, M.

Sacré, D. 252 Shasha, S.M. 233 Simonelli,R. 286 Smogorzewski, M. 233 Snelders, E.D. 252 Sonkodi, S. 387

Tabata, T. 382 Touwaide, A. 241

Wechsler,L. 340

Zuccoli, M. 274

Hadjiconstantinou, V. 214 Hazani, E. 233

KAIUiEH

E-Mail karger@karger.ch Fax+ 41613061234 http://www. karger. ch

(C) 1997 S. KargerAG, Basel

395 